\title{
Leber's hereditary optic neuropathy with diffuse white matter changes mimicking gliomatosis cerebri: illustrative case
}

Wakiko Saruta, MD, ${ }^{1}$ Ichiyo Shibahara, MD, PhD, ${ }^{1}$ Hajime Handa, MD, ${ }^{1}$ Madoka Inukai, MD, PhD, ${ }^{1}$ Shunsuke Kanayama, MD, ${ }^{2}$ Ryoma Yasumoto, ${ }^{3}$ Keizo Sakurai, ${ }^{3}$ Hisanao Akiyama, MD, PhD, ${ }^{4}$ Hitoshi Ishikawa, MD, PhD, ${ }^{2}$ Sumito Sato, MD, PhD, ${ }^{1}$ Takuichiro Hide, MD, PhD, ${ }^{1}$ and Toshihiro Kumabe, MD, $\mathrm{PhD}^{1}$

Departments of ${ }^{1}$ Neurosurgery and ${ }^{2}$ Ophthalmology, Kitasato University School of Medicine, Sagamihara, Kanagawa, Japan; ${ }^{3}$ Department of Clinical Laboratory, Kitasato University Hospital, Sagamihara, Kanagawa, Japan; and ${ }^{4}$ Department of Neurology, St. Marianna University School of Medicine, Kawasaki, Kanagawa, Japan

BACKGROUIND Leber's hereditary optic neuropathy (LHON) is a mitochondrial disease characterized by bilateral severe subacute central vision loss and a mutation in the mitochondrial DNA (mtDNA). The findings on cranial magnetic resonance imaging of patients with LHON vary from subtle to multiple white matter changes. However, they rarely present with diffuse infiltrative white matter changes.

OBSERVATIONS The authors reported a case with diffuse white matter changes mimicking gliomatosis cerebri (GC). The histological findings included only mild glial hyperplasia without immunohistochemical positivity, supporting the diagnosis of glial tumors. Analysis of mtDNA obtained from the blood and brain tissue revealed mutation of $\mathrm{m} .11778 \mathrm{G}>\mathrm{A}$ in the NADH dehydrogenase 4 gene, which confirmed the case as LHON. Immunohistochemistry of the brain tissue revealed 8-hydroxy-2'-deoxyguanosine positivity, suggesting the presence of oxidative stress.

LESSONS LHON is extremely difficult to diagnose unless one suspects or knows the disease. The present case brings attention not only to LHON but also to other mtDNA-mutated diseases that need to be considered with diffuse white matter changes or GC.

https://thejns.org/doi/abs/10.3171/CASE21161

KEYWORDS Leber's hereditary optic neuropathy; gliomatosis cerebri; mitochondrial disease; mitochondrial DNA

Leber's hereditary optic neuropathy (LHON) is a mitochondrial disease that predominantly affects men in their second and third decades of life. ${ }^{1}$ The clinical manifestation is a severe bilateral subacute central visual loss, with prevalence of approximately 1 in 10,000 to 50,000 cases. $^{2-5}$ The cause of LHON is a mutation at a maternally inherited mitochondrial DNA (mtDNA). Three mtDNA point mutations, such as m.11778G $>A$ in the NADH dehydrogenase (ND) 4 gene, m.3460G $>A$ in the ND1 gene, and m.14484T $>C$ in the ND6 gene, comprised more than $90 \%$ of LHON cases, ${ }^{5,6}$ and there are also rare variants, ${ }^{7}$ all affecting genes encoding complex I subunits of the mitochondrial respiratory chain. ${ }^{2,6}$ As a result, the dysfunction of the mitochondria damages retinal ganglion cells, which are highly susceptible to disrupted adenosine triphosphate production and oxidative stress. ${ }^{1}$
Gliomatosis cerebri (GC) is a rare disease with diffuse neoplastic glial infiltration involving more than two cerebral lobes. The 2007 World Health Organization (WHO) classification of tumors of the central nervous system categorized GC as a specific entity. The latest 2016 WHO classification excluded GC as a specific entity because DNA methylation revealed that GC corresponded to previously defined molecular subgroups of gliomas and did not demonstrate evidence for a distinct GC signature. ${ }^{8}$ However, we cannot ignore the invasive radiological features of GC, and further study is required to understand this unique tumor. ${ }^{9}$

We report a case that clinically and radiologically corresponded to the diagnosis of GC, with minimal or some findings of glial hyperplasia histologically. Immunohistochemical analyses did not show

ABBREVIATIONS ${ }^{18} \mathrm{~F}$-FDG $={ }^{18} \mathrm{~F}$-fluorodeoxyglucose; FLAIR = fluid-attenuated inversion recovery; GC = gliomatosis cerebri; LHON = Leber's hereditary optic neuropathy; $\mathrm{MRI}=$ magnetic resonance imaging; $\mathrm{mtDNA}=$ mitochondrial $\mathrm{DNA} ; \mathrm{ND}=\mathrm{NADH}$ dehydrogenase; $\mathrm{PCR}=$ polymerase chain reaction; $\mathrm{PET}=$ positron emission tomography; WHO = World Health Organization.

INCLUDE WHEN CITING Published June 28, 2021; DOI: 10.3171/CASE21161.

SUBMITTED March 17, 2021. ACCEPTED May 12, 2021.

(C) 2021 The authors, CC BY-NC-ND 4.0 (http://creativecommons.org/licenses/by-nc-nd/4.0/). 
positivity in IDH1R132H or TP53, but molecular analysis showed a point mutation at mtDNA of LHON. LHON is a disease primarily affecting the visual system, and it infrequently presents with small white matter lesions on brain magnetic resonance imaging (MRI). ${ }^{10}$ We discuss a case of LHON and other mtDNA-mutated diseases with diffuse radiological white matter changes mimicking $\mathrm{GC}$.

\section{Illustrative Case}

A 71-year-old man presented with bilateral vision loss that had been ongoing for 6 months. There was no relevant family history. He experienced progressive vision loss of the central visual field at the right eye and visited an ophthalmologist. MRI was performed to rule out diseases of the central nervous system resulting in diffuse white matter changes (Fig. 1A). Fluid-attenuated inversion-recovery (FLAIR) MRI showed a high-intensity area at the right temporal, occipital, and parietal lobes, the insula, the basal ganglia, the peduncle of the midbrain, the splenium of the corpus callosum, the optic chiasm, and the bilateral optic tracts (Fig. 1A). Gadolinium-enhanced T1-weighted MRI did not reveal an enhanced lesion (Fig. 1B). Radiological reports highly suggested GC. ${ }^{18} \mathrm{~F}$-fluorodeoxyglucose $\left({ }^{18} \mathrm{~F}-\mathrm{FDG}\right)$ positron emission tomography (PET) (Fig. 1C) did not reveal any uptake corresponding to this FLAIR highintensity lesion.

$\mathrm{He}$ then presented with progressive vision loss of the left eye. He was treated with intravenous steroid pulse therapy, but no improvement was noted after. There was no papilledema on fundoscopy, and the cerebrospinal fluid analysis was normal. Despite the diffuse white matter changes, the patient did not present with any other neurological symptoms except for bilateral vision loss. The ophthalmologists referred the patient to our department to confirm the histopathology of the brain.

A stereotactic biopsy was performed through the right parietal lobe to obtain tissue from the splenium of the corpus callosum (Fig. 1D). The histological findings revealed mild glial hyperplasia, but atypical cells or demyelination were not demonstrable (Fig. 2A and B). Immunohistochemical analyses showed negative IDH1R132H and TP53 and MIB1 labeling index $<1 \%$ (Fig. $2 \mathrm{C}, \mathrm{D}$, and $\mathrm{E}$, respectively); thus, there were no findings to support the diagnosis of GC or glial tumors. Because of the patient's progressive bilateral vision loss, the ophthalmologists suspected the possibility of LHON, resulting from the mtDNA mutation of m.11778G $>$ A extracted from the blood (Fig. 3).

We additionally investigated the brain tissue from the stereotactic biopsy, which also resulted in $\mathrm{m} .11778 \mathrm{G}>\mathrm{A}$ mtDNA mutation (Fig. 3). Because of the genetic result, the patient did not receive radiation and chemotherapy. We have been following the case for more than 2 years, and no radiological or neurological worsening has been noted (Fig. 1D). Additional immunohistochemistry using 8-hydroxy-2'-deoxyguanosine (8-OHdG, ab48505, Abcam) antibody demonstrated positivity (Fig. 2F), suggestive of cellular oxidative stress. mtDNA has a circular, doublestranded molecule with a length of $16,569 \mathrm{bp}$. Methods for detecting mtDNA mutations are as follows. Using polymerase chain reaction (PCR), we first amplified the whole length of mtDNA by three pairs of primers: Mito1_F: TTCCATCCACCCTCCTCTCCCTAG and Mito1_R: GAGCCCC ATTGTGTTGTGGTAAATA; Mito2_F: GTGGGCCTAGCCCTACTAGTCT CAA and Mito2_R: GGGATATTGATTTCACGGAGGATGG; and T27: TTCGCCCACTAAGCCAATCACTT and Mito3_R: GCGGGAGAAGTAGA TTGAAG ${ }^{11}$ After the confirmation of amplified PCR products and the purification step, we used the following three primers to detect the major three hotspot mtDNA mutations of LHON by Sanger sequencing: M3460P-1_F: TTGTACCCATTCTAATCGCAA; LEBER_SP_F: GGCGCAGTCATTCTC ATAAT; and m14484P-1_F: TCTTTCACCCACAGCACCAA.

\section{Discussion}

\section{Observations}

LHON is a relatively known disease among ophthalmologists; however, it is not as well known among most neurosurgeons and neurooncologists. The present case demonstrated diffuse infiltrative lesions on brain white matter and bilateral optic tracts. Approximately $67.9 \%$ of LHON cases present with high-intensity optic chiasm and bilateral optic tracts on T2-weighted MRI; ${ }^{12}$ however, $4.1 \%$
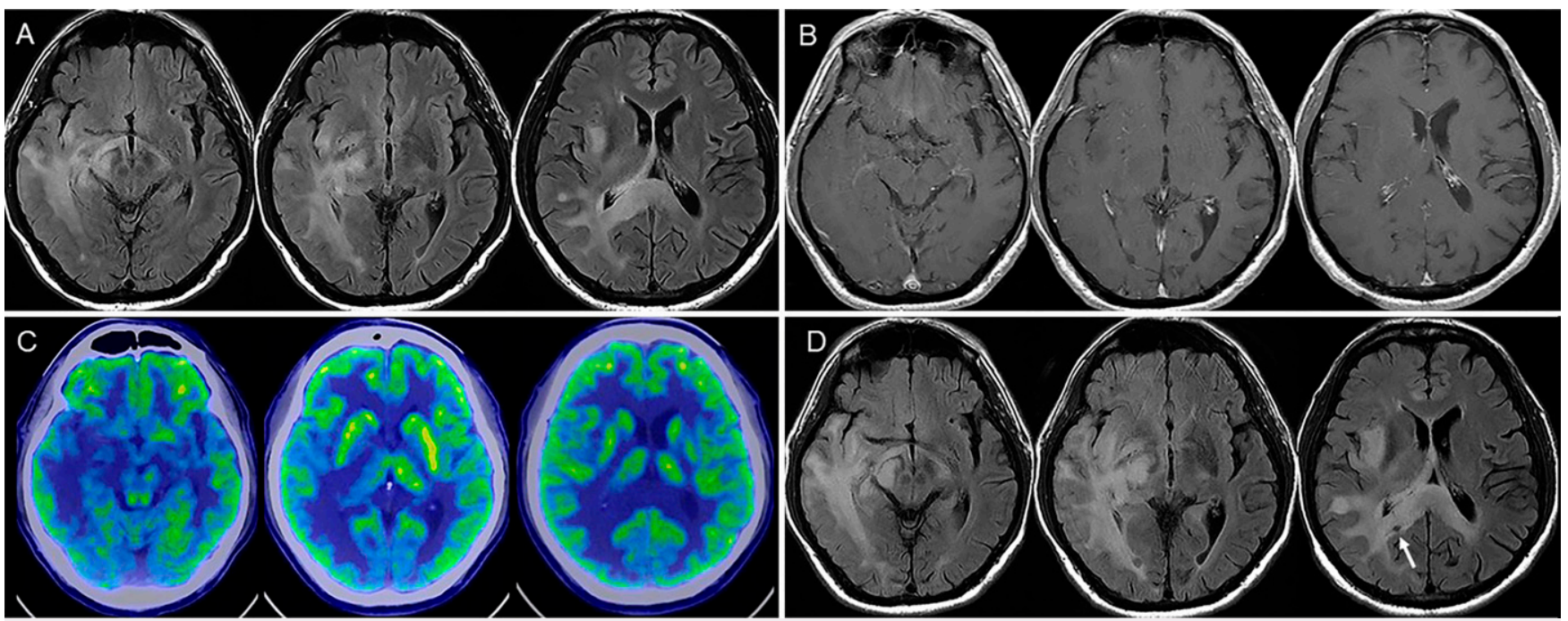

FIG. 1. A: FLAIR MRI shows a high-intensity area at the right temporal, occipital, and parietal lobes; right insula; basal ganglia; right peduncle of the midbrain; splenium of the corpus callosum; optic chiasm; and bilateral optic tracts. B: Gadolinium-enhanced T1-weighted MRI did not demonstrate an enhanced lesion. C: 18F-FDG PET did not have uptake corresponding to the FLAIR high-intensity lesion. D: FLAIR MRI demonstrates slight enlargement of high-intensity area at the splenium of the corpus callosum without any atrophic changes and clinical worsening. Arrow indicates the biopsied region as a small hole. 

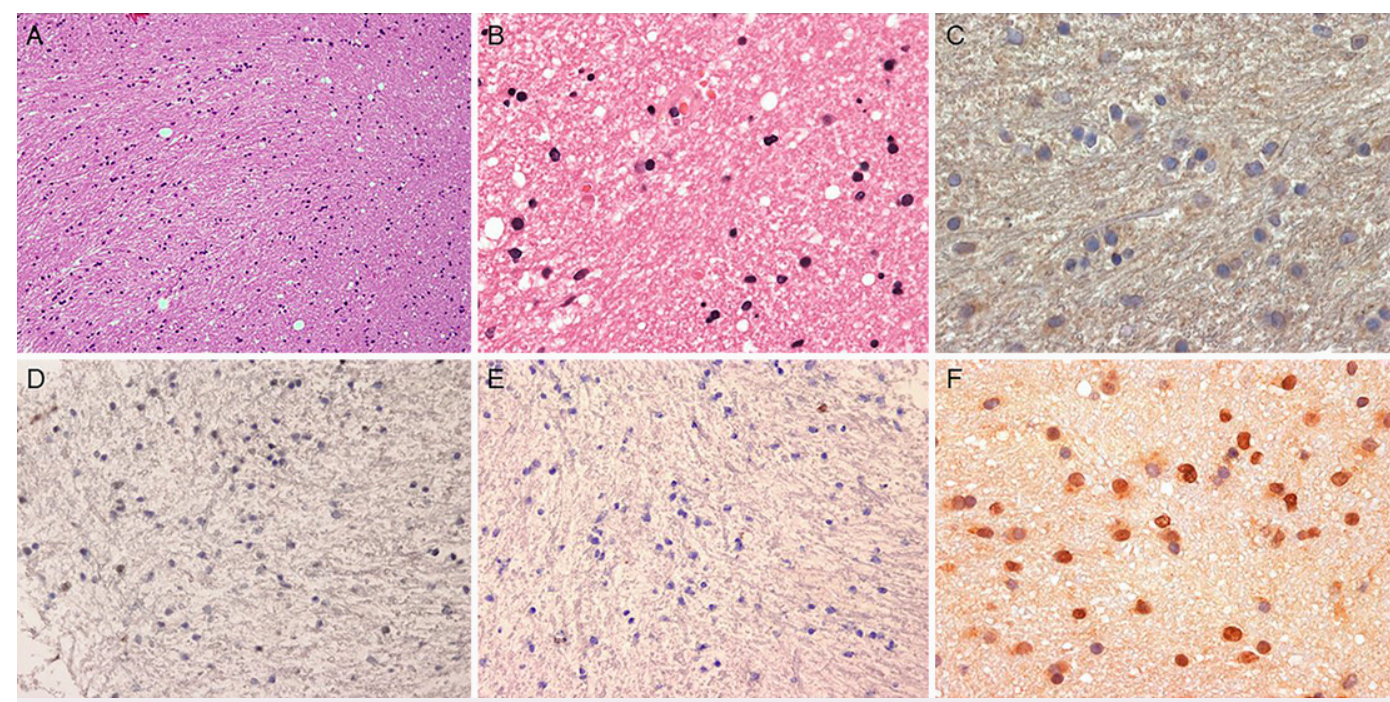

FIG. 2. Histological findings. A and B: Hematoxylin and eosin staining showing mild glial hyperplasia. C and D: Negative for immunohistochemical staining of IDH1R132H and TP53. E: MIB1 labeling index is less than 1\%. F: Positive for immunohistochemical staining of 8-hydroxy-2'-deoxyguanosine. (Original magnifications: A: $\times 100 ; B, C$, and F: $\times 400$; D and E: $\times 200$.)

to $10 \%$ of GC cases also present with such findings. ${ }^{13-15}$ Patients with LHON infrequently present with white matter changes on MRI, ${ }^{10,16,17}$ but none so far have presented with diffuse infiltrates, such as the present case.

Patients with LHON showed significantly reduced volume of the optic chiasm, optic tracts, white matter in the optic radiation, and other subcortical brain structures, which could be detected using diffusion tensor MRI, $3 \mathrm{~T} \mathrm{MRI}$, or $7 \mathrm{~T} \mathrm{MRI}{ }^{18-22}$ However, such studies assessed patients with LHON with disease duration over 10 years, ${ }^{18,22}$ indicating that atrophic changes would appear in a chronic phase. Case reports demonstrated chronological changes from swollen to atrophic optic tracts over time ${ }^{23}$ therefore, a swollen structure may be an initial change in LHON. The present case had diffuse white matter changes with thickening of the splenium of the corpus callosum. Thickened splenium has never been reported in patients with LHON and may indicate glioma-like change, but the tissue obtained from the splenium was negative for histological features of gliomas. We presume that the splenium of the corpus callosum will be atrophic in a later phase during follow-up. LHON-related genetic mutations causing mitochondrial dysfunction can induce occult white matter changes in the brain. ${ }^{20}$ Because LHON is a disease in young adults, older patients with longer incubation time will transform the occult white matter changes to visible changes.

Epidemiologically, neuroepithelial tumors, including all grades of gliomas, are more frequently observed in men. ${ }^{24,25}$ In terms of survival, male patients with glioblastoma ${ }^{24,26-28}$ and grade 3 gliomas $^{29}$ present with shorter survival than female patients. Therefore, although still controversial, ${ }^{28,30}$ male patients with malignant gliomas had worse survival. As with other gliomas, GC is also frequently observed in men. ${ }^{13,31-34}$ Contrary to other malignant gliomas, two studies showed that male patients with GC have more prolonged overall survival. ${ }^{32,35}$ In these studies, $36.4 \%$ to $39.5 \%$ of the GC cases were diagnosed based on clinical and radiological methods alone without a histological diagnosis. On the contrary, other studies demonstrated no survival difference between sexes in patients with GC, ${ }^{13,33}$ all of whom had histologically confirmed GC. As our case presented, a male preponderance nonmalignant disease with diffuse white matter changes can be misdiagnosed as GC, possibly explaining the prolonged survival in men. Although radiological findings are typical for GC, a histological analysis should be conducted to rule out LHON.

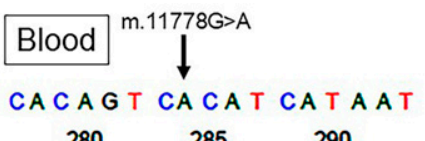

$280 \quad 285 \quad 290$

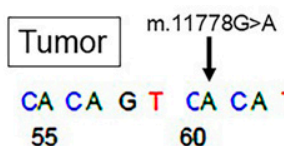

$60 \quad 65$

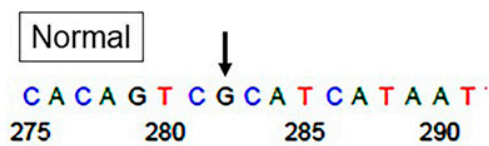

290

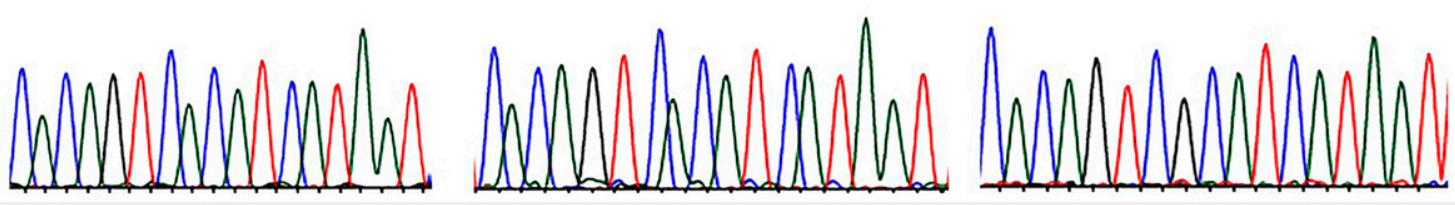

FIG. 3. The result of Sanger sequencing from the blood, brain tissue, and reference as a normal control (arrows). mtDNA from the blood and brain tissue showed $\mathrm{m} .11778 \mathrm{G}>$ A mutation. 
We assessed the tumor tissue obtained from brain biopsy to investigate for $\mathrm{mtDNA}$ mutation. Because $\mathrm{m} .11778 \mathrm{G}>\mathrm{A}$ mutation was detected from the mtDNA of the blood, the same mutation was detected from the mtDNA of the brain. To assess the mitochondrial dysfunction, we immunohistochemically evaluated 8-OHdG, a marker of cellular oxidative stress. ${ }^{36}$ Corresponding to the previous report with increased 8-OHdG from leukocyte DNA of patients with LHON, the brain tissue showed positivity for 8-OHdG antibody, indicating the presence of oxidative stress in the brain. Kovács et al. also histologically demonstrated oxidative stress in the brain white matter of a patient with LHON as upregulation of mitochondrial manganese superoxide dismutase but used autopsy cases. ${ }^{37}$ LHON is a disease of the visual system in which histological assessment is difficult; thus, the histological findings of the present case were essential to understand the pathophysiology of the disease. In addition to the three major mtDNA mutations of LHON (m.11778G $>A$, $\mathrm{m} .3460 \mathrm{G}>\mathrm{A}$, and $\mathrm{m} .14484 \mathrm{~T}>\mathrm{C}$ ), 46-point mutations have been reported as mtDNA mutations in patients with $\mathrm{LHON}{ }^{7}$ Most of them encode mtDNA genes of ND4L, ND4, ND5, and ND6. Yeung et al. analyzed the mtDNA in glioblastoma and found that ND4L, ND4, ND5, and ND6 were also frequently mutated in mtDNA. ${ }^{38}$ LHON and glioblastoma presented with the same mutated genes, but they did not share the mutated reference positions. Unlike other mitochondrial diseases, LHON shared mutated genes with glioblastoma, genetically potentiating LHON to mimic GC.

\section{Lessons}

We presented a case of LHON with diffuse white matter changes mimicking GC. Histological diagnosis is essential to exclude the possibility of GC but is not enough to diagnose LHON. Without the detection of mtDNA mutation of $\mathrm{m} .11778 \mathrm{G}>\mathrm{A}$, it would have been difficult to exclude the diagnosis of GC and possibly would have led to unnecessary treatment involving radiation and chemotherapy. Our case suggested that diffuse white matter changes in a man should be differentially diagnosed as LHON or mitochondrial diseases, which can be confirmed with mtDNA mutation findings.

\section{Acknowledgments}

We would like to thank Editage for English language editing.

\section{References}

1. Meyerson C, Van Stavern G, McClelland C. Leber hereditary optic neuropathy: current perspectives. Clin Ophthalmol. 2015;9: 1165-1176.

2. Yu-Wai-Man P, Turnbull DM, Chinnery PF. Leber hereditary optic neuropathy. J Med Genet. 2002;39(3):162-169.

3. Perez F, Anne O, Debruxelles S, et al. Leber's optic neuropathy associated with disseminated white matter disease: a case report and review. Clin Neurol Neurosurg. 2009;111(1):83-86.

4. Puomila A, Hämäläinen P, Kivioja S, et al. Epidemiology and penetrance of Leber hereditary optic neuropathy in Finland. Eur J Hum Genet. 2007:15(10):1079-1089.

5. Yu-Wai-Man P, Griffiths PG, Chinnery PF. Mitochondrial optic neuropathies-disease mechanisms and therapeutic strategies. Prog Retin Eye Res. 2011;30(2):81-114.

6. Kirches E. LHON: mitochondrial mutations and ore. Curr Genomics. 2011;12(1):44-54

7. Dai $Y$, Wang $C$, Nie Z, et al. Mutation analysis of Leber's hereditary optic neuropathy using a multi-gene panel. Biomed Rep. 2018;8(1): 51-58.
8. Herrlinger U, Jones DTW, Glas M, et al. Gliomatosis cerebri: no evidence for a separate brain tumor entity. Acta Neuropathol. 2016; 131(2):309-319.

9. Morales La Madrid A, Ranjan S, Warren KE. Gliomatosis cerebri: a consensus summary report from the Second International Gliomatosis Cerebri Group Meeting, June 22-23, 2017, Bethesda, USA. J Neurooncol. 2018;140(1):1-4.

10. Matthews L, Enzinger C, Fazekas F, et al. MRI in Leber's hereditary optic neuropathy: the relationship to multiple sclerosis. J Neurol Neurosurg Psychiatry. 2015;86(5):537-542.

11. Akanuma J, Muraki K, Komaki H, et al. Two pathogenic point mutations exist in the authentic mitochondrial genome, not in the nuclear pseudogene. J Hum Genet. 2000;45(6):337-341.

12. Blanc $C$, Heran $F$, Habas $C$, et al. MRI of the optic nerves and chiasm in patients with Leber hereditary optic neuropathy. J Neuroophthalmol. 2018;38(4):434-437.

13. Georgakis MK, Tsivgoulis G, Spinos D, et al. Clinical, neuroimaging and histopathological features of gliomatosis cerebri: a systematic review based on synthesis of published individual patient data. J Neurooncol. 2018;140(2):467-475.

14. Traynis I, Singer S, Winterkorn J, et al. Infiltration of the optic chiasm, nerve, and disc by gliomatosis cerebri. J Neuroophthalmol. 2014;34(1):44-46.

15. Peretti-Viton $\mathrm{P}$, Brunel $\mathrm{H}$, Chinot $\mathrm{O}$, et al. Histological and MR correlations in gliomatosis cerebri. J Neurooncol. 2002;59(3): 249-259.

16. Jančić J, Dejanović I, Radovanović S, et al. White matter changes in two Leber's hereditary optic neuropathy pedigrees: 12-year follow-up. Ophthalmologica. 2016;235(1):49-56.

17. Mercuri MA, White $H$, Oliveira $C$. Vision loss and symmetric basal ganglia lesions in Leber hereditary optic neuropathy. $J$ Neuroophthalmol. 2017:37(4):411-413.

18. Jonak K, Krukow P, Symms M, et al. Neuroanatomical changes in Leber's hereditary optic neuropathy: clinical application of 7T MRI submillimeter morphometry. Brain Sci. 2020;10(6):359.

19. Milesi J, Rocca MA, Bianchi-Marzoli S, et al. Patterns of white matter diffusivity abnormalities in Leber's hereditary optic neuropathy: a tractbased spatial statistics study. J Neurol. 2012;259(9):1801-1807.

20. Long $M$, Wang $L$, Tian $Q$, et al. Brain white matter changes in asymptomatic carriers of Leber's hereditary optic neuropathy. J Neurol. 2019;266(6):1474-1480.

21. Barcella V, Rocca MA, Bianchi-Marzoli S, et al. Evidence for retrochiasmatic tissue loss in Leber's hereditary optic neuropathy. Hum Brain Mapp. 2010;31(12):1900-1906.

22. Jonak K, Krukow P, Karakuła-Juchnowicz H, et al. Aberrant structural network architecture in Leber's hereditary optic neuropathy. Minimum spanning tree graph analysis application into diffusion 7T MRI. Neuroscience. 2021;455:128-140.

23. van Westen D, Hammar B, Bynke $G$. Magnetic resonance findings in the pregeniculate visual pathways in Leber hereditary optic neuropathy. J Neuroophthalmol. 2011;31(1):48-51.

24. Le Rhun E, Weller M. Sex-specific aspects of epidemiology, molecular genetics and outcome: primary brain tumours. ESMO Open. 2020;5(suppl 4):e001034.

25. Ostrom QT, Cioffi G, Gittleman H, et al. CBTRUS statistical report: primary brain and other central nervous system tumors diagnosed in the United States in 2012-2016. Neuro Oncol. 2019;21(suppl 5):v1-v100.

26. Yang W, Warrington NM, Taylor SJ, et al. Sex differences in GBM revealed by analysis of patient imaging, transcriptome, and survival data. Sci Transl Med. 2019;11(473):eaao5253.

27. Ostrom QT, Rubin JB, Lathia JD, et al. Females have the survival advantage in glioblastoma. Neuro Oncol. 2018;20(4):576-577.

28. Gittleman H, Ostrom QT, Stetson LC, et al. Sex is an important prognostic factor for glioblastoma but not for nonglioblastoma. Neurooncol Pract. 2019;6(6):451-462. 
29. Fang JH, Lin DD, Deng XY, et al. Epidemiological trends, relative survival, and prognosis risk factors of WHO Grade III gliomas: a population-based study. Cancer Med. 2019;8(6): 3286-3295.

30. Carstam L, Rydén I, Gulati S, et al. Socioeconomic factors affect treatment delivery for patients with low grade glioma: a Swedish population-based study. J Neurooncol. 2020;146(2):329-337.

31. Chen S, Tanaka S, Giannini C, et al. Gliomatosis cerebri: clinical characteristics, management, and outcomes. J Neurooncol. 2013;112(2):267-275.

32. Georgakis MK, Spinos D, Pourtsidis A, et al. Incidence and survival of gliomatosis cerebri: a population-based cancer registration study. $J$ Neurooncol. 2018;138(2):341-349.

33. Georgakis MK, Tsivgoulis G, Spinos D, et al. Prognostic factors and survival of gliomatosis cerebri: a systematic review and metaanalysis. World Neurosurg. 2018;120:e818-e854.

34. Khalafallah AM, Kopparapu S, Mukherjee D. Predictive factors for overall survival in surgical cases of gliomatosis cerebri from the National Cancer Database. J Clin Neurosci. 2020;81:186-191.

35. Taillibert S, Chodkiewicz C, Laigle-Donadey F, et al. Gliomatosis cerebri: a review of 296 cases from the ANOCEF database and the literature. J Neurooncol. 2006;76(2):201-205.

36. Kasai $\mathrm{H}$. Analysis of a form of oxidative DNA damage, 8-hydroxy2'-deoxyguanosine, as a marker of cellular oxidative stress during carcinogenesis. Mutat Res. 1997;387(3):147-163.
37. Kovács GG, Höftberger R, Majtényi K, et al. Neuropathology of white matter disease in Leber's hereditary optic neuropathy. Brain. 2005;128(Pt 1):35-41.

38. Yeung KY, Dickinson A, Donoghue JF, et al. The identification of mitochondrial DNA variants in glioblastoma multiforme. Acta Neuropathol Commun. 2014;2(1):1.

\section{Disclosures}

The authors report no conflict of interest concerning the materials or methods used in this study or the findings specified in this paper.

\section{Author Contributions}

Conception and design: Shibahara, Saruta. Acquisition of data: Shibahara, Saruta, Kanayama, Yasumoto, Akiyama, Ishikawa, Sato. Analysis and interpretation of data: Shibahara, Saruta, Yasumoto, Sakurai. Drafting the article: Shibahara, Saruta, Hide. Critically revising the article: Shibahara, Saruta, Kumabe. Reviewed submitted version of manuscript: Shibahara, Saruta, Akiyama, Kumabe. Approved the final version of the manuscript on behalf of all authors: Shibahara. Statistical analysis: Saruta. Administrative/technical/material support: Saruta, Handa, Inukai. Study supervision: Shibahara, Saruta, Kumabe.

\section{Correspondence}

Ichiyo Shibahara: Kitasato University School of Medicine, Kanagawa, Japan. shibahar@med.kitasato-u.ac.jp. 